\title{
A Comparative Analysis for Shallow Aquatic Images Visibility Improvement and Restoration
}

\author{
Gaurav Goel \\ Assistant Professor, IT Deptt, \\ MIET, Meerut
}

\author{
Praveen Mishra \\ Assistant Professor, CSE Deptt, \\ BIT, Meerut
}

\author{
Pooja Agarwal \\ Assistant Professor, MCA Deptt, \\ MIET, Meerut
}

\begin{abstract}
This paper proposes an comparative analysis for shallow aquatic images visibility improvement which firstly classify whether the image is clear or haze image, along with the identification of more or less haze. The paper presented a separate method for low and high haze content image which utilizes a combination of dark channel prior, median filter, histogram equalization, gamma correction, dark channel with morphological action, in order to attain effective haze reduction and circumvent halo effects in complex structure single image. Our scheme, also lookup the effect of an adaptive gamma correction method for additional improvement of transmission depth which enables a important enrichment over existing state-of-the-art schemes based on dark channel prior.
\end{abstract}

\section{Keywords}

Undersea image, DCP, Transmission Depth, Adaptive Gamma Correction, Histogram Equalization.

\section{INTRODUCTION}

The image captured in outdoor scene are highly despoiled due to poor lighting situation or due to turbid medium in poor weather, such as haze, water droplets, dust particles or due to submergence in water. So due to these particles the irradiance coming from the object is scattered or absorbed between the digital camera and the captured object. It produces an effect called haze, which trim down the overall contrast in acquired images and basis of color change, openly affecting the visibility of image.

In contrast to common photographs, underwater optical images suffer from poor visibility owing to the medium, which causes mainly scattering and absorption. Large suspended particles cause scattering, as in fog or turbid water that contains abundant particles. Color distortion originates from its inherent optical properties encountered by light traveling in water at different wavelengths, causing ambient underwater environments to be dominated by a bluish tone.

In addition, absorption substantially reduces the light energy. The random attenuation of light primarily causes a hazy appearance, while the fraction of light scattered back from the water along the line of sight considerably degrades the scene contrast.

As far as single image method are newly arose, He et al. [1], [2] proposed a fresh method called Dark Channel Prior (DCP). Their technique is based on the analysis that fog-free images have at least single color channel in the RGB band with little intensity value. More newly, the DCP method has also been useful in underwater image restoration [6], [7], [10], [11]. However, those methods do not deal with some of the important DCP restrictions and the required alteration in the assumptions for underwater imaging system. Thus, the key input of the present work is the modification of the DCP in order to prevail over its boundaries for applications in waterlogged imaging system.
The study [31], [32] highlights the comparative review of approaches for outdoor hazy, underwater and night images visibility enhancement and restoration. The approach for shallow underwater image visibility and color improvement using morphological operation reduce amount of halo effect [30] in image.

\section{AQUATIC IMAGE FORMATION MODEL}

According to [1], the formation of a haze image is:

$\operatorname{img}(p)=j \operatorname{jrad}(p) \cdot \operatorname{tmap}(p)+B(1-\operatorname{tmap}(p))$

where jrad is the scene radiance, img is the observed intensity, $B$ is the global atmospheric light, and tmap(p) is the transmission map. Underwater image improvement in [6], [7], [8] is done using this model. Because Both day and underwater images are despoiled by the turbid medium, an underwater image is similar to a day haze image and the intensity is being composed of two components: the indirect transmission due to scattering by the medium dirt particles and the direct transmission of light from the scene.

Because of dissimilarity in attenuation between water and atmospheric environment, one can not equate the underwater imaging model to its atmospheric version. The model for underwater images [8] can be described mathematically after considering both the effect of absorption and scattering on light attenuation as:

The airlight constant $\mathrm{B}$ is predictable by finding the brightest pixel in the underwater dark channel [1]. The value of local patch is defined as $\Omega(p)=15^{*} 15$ and the transmission tmap $(p)$ [8] is calculated as :

$\operatorname{tmap}_{\beta}^{c}(p)=1-\operatorname{tmap}_{\alpha}^{c}(p)=$
$1-\min _{y \varepsilon \Omega(p)}\left(\min _{c \varepsilon g, b} \frac{I m g^{c}(y)}{B^{c}}\right)$

where Img is the observed intensity, $\mathrm{c}$ is a color channel (red, green or blue), B is the scattering light called as background light, tmap $\alpha$ is the scattering rate and $\operatorname{tmap} \beta$ is the transmission (Portion of the scene radiance reaching the camera).

\section{AQUATIC IMAGES VISIBILITY AND COLOR CAST ENHANCEMENT ALGORITHM}

1) Firstly obtain \& split the sample undersea input RGB images.

2) Classify whether the obtained image is clear or haze in appearance.

If $($ Mean $>\sigma)$ Then

i. Print "Image is Haze Image"

ii. Execute the step 3. 
Else

i. Print "Image is Clear Image"

3) Classify whether the input image have large or fewer amount of haze .

If $($ Mean $>\lambda)$ Then

i. Print "Image is More Haze"

ii. $\quad$ Execute the step 4 to step 13.

Else

i. Print "Image is Less Haze"

ii. Apply dark channel prior directly on input image with morphological operation.

iii. Use median filter with adaptive gamma correction.

iv. Estimate refines transmission \& recovers the scene radiance.

4) Use histogram equalization technique for improvement in image visibility.
5) Utilize gamma correction technique for adjusting range of color ratio.

6) Employ the dark channel prior method on preprocessed input image.

7) Apply morphological action to reduce amount of halo effect from image.

8) Guesstimate the background radiance using brightest pixel in dark channel.

9) Estimate the better transmission map using above improve dark channel.

10) Extract the scene radiance using morphological better transmission.

11) Enrich the transmission depth using soft matting \& obtain sight radiance.

12) Boost the transmission depth using adaptive gamma correction method.

13) Obtained the final scene radiance using above boosted transmission depth.

\section{PROPOSED SCHEME FLOWCHART \& MODULES DESCRIPTION}

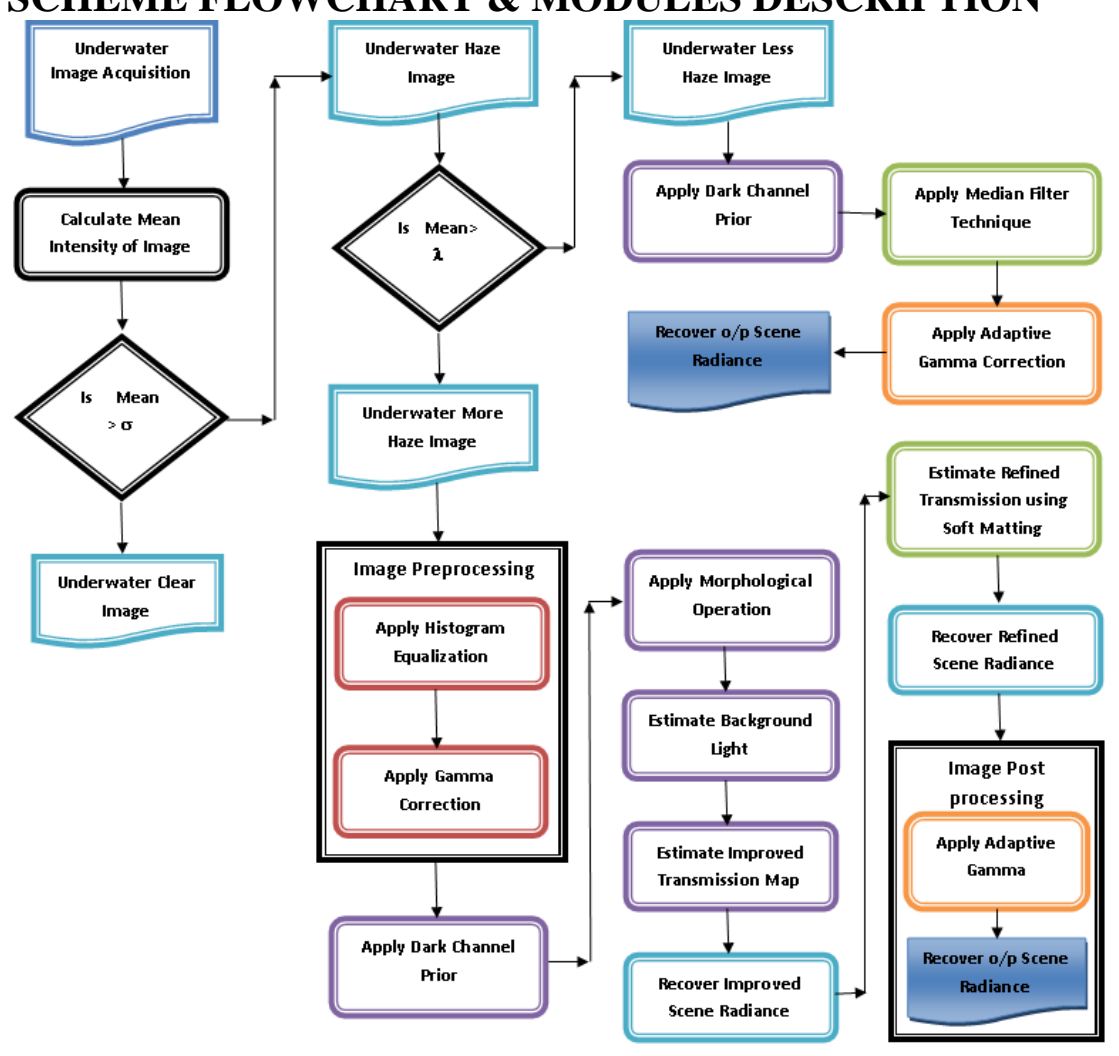

The model proposes a visibility improvement and color rectification method that uses a combination of four major modules:

\section{1) Haze Classification \& Identification}

Module: After the acquisition of underwater image, the intensity of image is calculated and is compared with the threshold value obtains after averaging the mean intensities of several clear \& haze images [32]. If the intensity is greater than the set threshold the image is consider to be haze otherwise clear image.
The image is then check to know whether the image is more or less haze. If more haze image is found the modules second, third \& fourth are executed to obtain the clear image otherwise the input image is directly pass through dark channel prior with morphological operation [30], median filter $\&$ adaptive gamma correction (Module 4) to extract the clear image. The equation given by [14] used to preserve edge information is shown below:

$$
e(p)=\omega\left(\min _{c}\left(\operatorname{median}_{y \leq n(p)} J(y)\right)\right)-\min _{y s \Omega(p)} J(y)
$$


2) Image Preprocessing Module: When the Red, Green, and Blue channels of an image are not properly balanced the trouble of color cast occurs. The color cast can be across the entire range of pixel values or can bound itself to the underline, shade of the image. Our proposed technique has solved this problem by applying visibility improvement and contrast correction method [12]. This is processed out by elongating the range of intensity values in order to cover the preferred range of values. Prior to performing contrast variation a step is made for the higher and lower limits of the image for contrast modification of each band. Usually, the range of values in 8 bit color channel is $0-255$.

Our proposed method has use a common normalization scheme to find the slightest and the majority pixel values in the histogram and stretch them between the particular ranges using histogram equalization which in turn recover the visibility of image and gamma correction technique to progress the contrast of an image [30], [33]

3) Scene Radiance Recovery Module: The visibility of undersea image is spoil due to occurrence of haze. The dark channel prior provides a way by which to approximate better transmission in an underwater image. Here the dark channel of image is recognized first and then used for estimation of the background light $\mathrm{B}$ in the submerged image. In our approach the brightest 0.1 percent of pixels will chosen from within the dark channel prior. From among these, the pixels with the peak intensity in the input image are determined to be the background light B. The value of local patch is defined as $\Omega(p)=15 * 15$.

The dark channel obtain by old dark channel prior system contain lot more halo effect which lead to blocky transmission map estimation \& scene radiance recovery. For this cause, our approach proposed a look up transmission procedure which uses a morphological technique to lessen generation of halo effects [30]. The transmission is estimates as [1]

$t(p)=1-\omega \min _{y \sin (p)}\left(\min _{\varepsilon} \frac{I^{v}(y)}{B^{c}}\right)$

Because the prime operation of the dark channel prior depends on the minimum filter, the transmission map will usually practice a loss of edge information when estimation occurs. For this reason, our method used a refined transmission procedure which uses a soft matting technique [2] to eradicate blocky artifacts and preserve edge information of input submerged images.

$I(p)=\frac{I(p)-B}{\max \left(t(p), t_{s}\right)}+B$

4) Image Post processing Module: The dark channel prior depends on the least value of the RGB channel and always produces an inadequate transmission map for images captured during underwater conditions. This is because the intensity value will be minor for at least one RGB channel in underwater images [31]. In order to attain optimum haze removal results and to guesstimate efficient transmission map, our proposed method has apply an adaptive gamma correction technique to alter the intensity of the transmission map during this course of action. Adaptive gamma correction technique [14] redistribute within the dynamic range of the histogram by using a changeable adaptive parameter. $t_{\text {mew }}(p)=\left(p_{\max }\right)\left(\frac{t(p)}{p_{\max }}\right)^{P}$

\section{EXPERIMENTAL OBSERVATIONS}

The presented algorithm is implemented in MATLAB executing on operating system Windows XP. The PSNR (peak to signal ratio) \& CNR (contrast to noise ratio) has been estimated for diverse output images of different scheme using matlab code.

Table 1: (Sample input images)

\begin{tabular}{|c|c|c|c|}
\hline \multicolumn{4}{|c|}{ Sample Input Images } \\
\hline $\begin{array}{c}\text { S.N } \\
\boldsymbol{o}\end{array}$ & Image Name & Image & $\begin{array}{c}\text { Original } \\
\text { CNR }\end{array}$ \\
\hline 1 & Input1.jpg & & 252.064 \\
\hline 2 & Input2.jpg & & 287.168 \\
\hline
\end{tabular}

Table2: For More Haze Image

(PSNR \& CNR values for first output image)

\begin{tabular}{|c|c|c|c|c|}
\hline \multicolumn{5}{|c|}{ RGB Input Image 1 } \\
\hline $\begin{array}{c}\text { S. } \\
\text { No }\end{array}$ & Output Image & $\begin{array}{c}\text { Proposed } \\
\text { Output }\end{array}$ & PSNR & CNR \\
\hline 1 & Output 1 & 13.956 & 318.673 \\
\hline 2 & & & & \\
\hline 3 & & Output 2 & $\mathbf{1 4 . 2 5 2}$ & $\mathbf{3 0 1 . 6 9 8}$ \\
& & & & \\
\hline & & Output 3 & 14.336 & 302.560 \\
\hline
\end{tabular}

Table 3: For Less Haze Image

(PSNR \& CNR values for second output image)

\begin{tabular}{|c|c|c|c|c|}
\hline \multicolumn{5}{|c|}{ RGB Input Image 2 } \\
\hline S.No & Image & Approach & PSNR & CNR \\
\hline 1 & & $\begin{array}{c}\text { Dark } \\
\text { Channel } \\
\text { Prior }\end{array}$ & 24.867 & 314.949 \\
\hline 2 & & $\begin{array}{c}\text { Median } \\
\text { Filter }\end{array}$ & 24.858 & 307.892 \\
\hline
\end{tabular}




\begin{tabular}{|c|c|c|c|c|}
\hline 3 & Sestive & $\begin{array}{c}\text { Adaptive } \\
\text { Gamma } \\
\text { Correction }\end{array}$ & $\mathbf{2 7 . 5 5 1}$ & $\mathbf{3 0 0 . 9 0 3}$ \\
\hline
\end{tabular}

Table 4: (PSNR \& CNR based comparison of different methods)

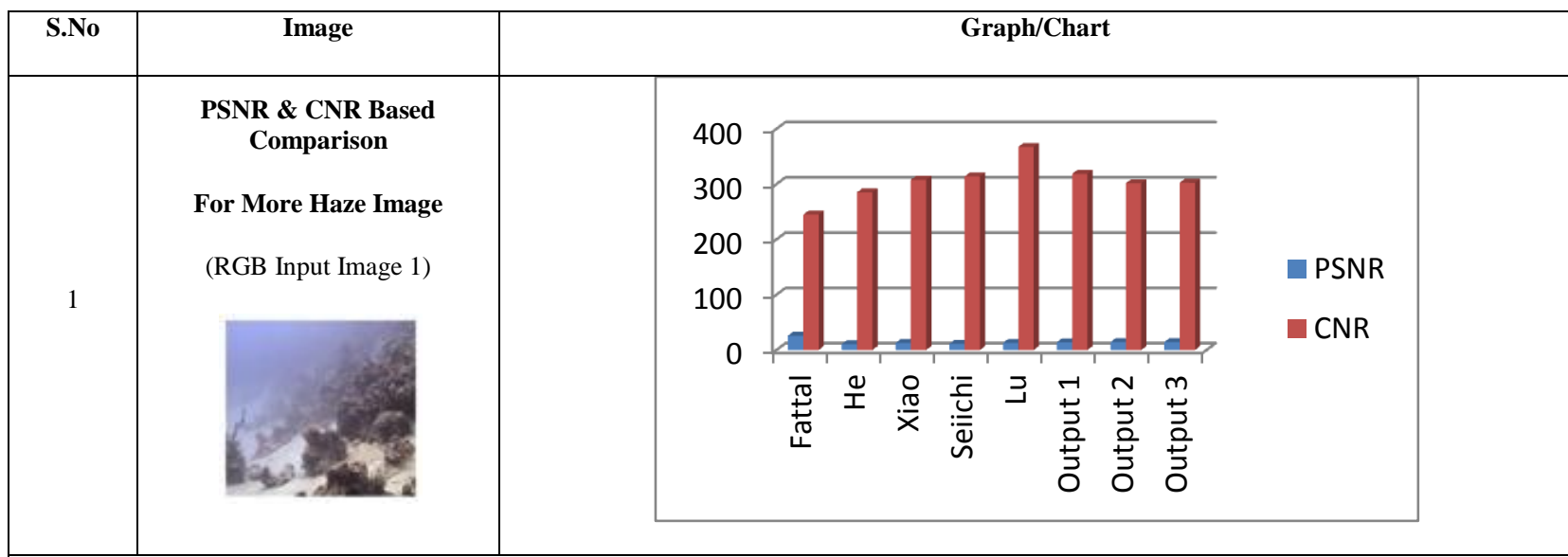

The above graphs showed that proposed method produced better PSNR values from all other authors like He, Xiao, Seiichi, Lu except the result of Fattal as well as better CNR values from Fattal, He, and nearby to all other authors for first input image in case of more haze.

\begin{tabular}{|l|c|c|c|}
\hline $\begin{array}{c}\text { PSNR \& CNR Based } \\
\text { Comparison }\end{array}$ & $\begin{array}{l}\text { For Less Haze Image } \\
\text { (RGB Input Image2) }\end{array}$ \\
The above graphs showed that proposed method produced better PSNR values from all other authors like Fattal, He and Hung as \\
well as better CNR values from all other authors for second input image in case of less haze.
\end{tabular}

\section{DISCUSSION \& CONCLUSION}

Our proposed paper have tried to compare the effect of shallow aquatic images visibility improvement methods via image enhancement and color correction method such as median filter, histogram equalization, gamma correction technique and dark channel prior.

Our scheme have also use morphological action on dark channel, along with soft matting \& adaptive gamma correction technique for getting better image visual quality such as, reduce halo effect, improve and filter transmission estimation procedure, improving image color contrast as well as dropping noise and artifacts from obtained output image which enables a important enrichment over existing state-of-the-art scheme based on dark channel prior.

\section{REFERENCES}

[1] K. He, J. Sun, and X. Tang. "Single Image Haze Removal Using Dark Channel Prior," in Proc. IEEE Conf.
Computer Vision and Pattern Recognition. Miami, FL, pp. 1956-1963, June 2009.

[2] Kaiming He, Jian Sun, and Xiaoou Tang." Single Image Haze Removal Using Dark Channel Prior". IEEE Transaction on Pattern Analysis and Machine Intelligence, VOL. 33, NO. 12, December 2011.

[3] W. J. Wang, B. H. Chen, and S. C. Huang, "A Novel Visibility Restoration Algorithm for Single Hazy Images," IEEE Int. Conf. on Systems, Man, and Cybernetics, October 2013.

[4] Muhammad Suzuri Hitam, Ezmahamrul Afreen Awalludin "Mixture contrast limited adaptive histogram equalization for underwater image enhancement" IEEE Int. Conf. on Computer Application Technology (ICCAT), pp. 1-5, January 2013.

[5] Deepak Kumar Naik, Deepak Kumar Rout "Outdoor image enhancement: Increasing visibility under extreme haze and lighting condition" IEEE Int. Advance 
Computing Conference (IACC), pp. 1081-1086, February 2014.

[6] H. Yang, P. Chen, C. Huang, Y. Zhuang and Y. Shiau, "Low complexity underwater image enhancement based on dark channel prior," IEEE II Int. Conf. on Innovation in Bio-Inspired Computing and Application (IBICA), pp. 17-20, December 2011

[7] P. Drews-jr, E. do Nascimento, F. Moraes, S. Botelho, M. Campos "Transmission estimation in underwater single images" IEEE Int. Conf. on computer vision workshops (ICCVW), pp. 825-830, December 2013

[8] Haocheng Wen, Yonghong Tian, Tiejun Huang, Wen Gao "Single underwater image enhancement with a new optical model" IEEE Int. Symposium on Circuits and Systems (ISCAS), pp. 753-756, May 2013.

[9] J.Y. Chiang and Y.C. Chen, "Underwater image enhancement by wavelength compensation and dehazing", IEEE Transactions on Image Processing, vol.21, no.4, pp.1756-1769, 2012.

[10] Huimin Lu, Seiichi Serikawa "A novel underwater scene reconstruction method", IEEE International Symposium on Computer, Consumer and Control (IS3C), pp. 773775, June 2014.

[11] Huimin Lu, Seiichi Serikawa "Underwater scene enhancement using weighted guided median filter", IEEE Int. Conf on Multimedia and Expo (ICME), pp. 773-775, July 2014.

[12] Sos Agaian, Mehdi Roopaei "New haze removal scheme and novel measure of enhancement" IEEE Int. Conf. on Cybernetics (CYBCONF) , pp. 219-224, June 2013.

[13] H. Lu, Y. Li, S. Serikawa, "Underwater image enhancement using guided trigonometric bilateral filter and fast automation color correction", in: Proc. of 20th IEEE International Conference on Image Processing (ICIP ‘13), pp.3412-3416, September 2013.

[14] Shih-Chia Huang, Bo-Hao Chen and Wei-Jheng Wang “ Visibility restoration of single hazy images captured in real world weather conditions" IEEE Trans circuits and systems for video technology, 2014.

[15] R. Schettini and S. Corchs, "Underwater image processing: state of the art of restoration and image enhancement methods," EURASIP (A spriger open journal) Journal on Advances in Signal Processing, 746052, April 2010

[16] L. Chao and M. Wang, "Removal of water scattering," IEEE Int. Conf. on Computer Engineering and Technology (ICCET), vol. 2, pp. 35-39, April 2010

[17] Kashif Iqbal, Rosalina Abdul Salam "Enhancing the low quality images using unsupervised colour correction method", IEEE Int. Conf. on System, Man and Cybernetics (SMC), pp. 1703-1709, October 2010.

[18] Luz Abril Torres-Méndez and Gregory Dudek, "Color Correction of Underwater Images for Aquatic Robot Inspection" Lecture Notes in Computer Science 3757, Springer A. Rangarajan, B.C Vemuri, A.L. Yuille (Eds.), pp. 60-73, ISBN:3-540- 30287-5,2005.

[19] J. Jaffe. "Computer modeling and the design of optimal underwater imaging systems". IEEE Journal of Oceanic Engineering (JOE), 15(2):101-111, April 1990.
[20] B. McGlamery. "A computer model for underwater camera systems". In SPIE 0208, Ocean Optics VI, volume 208, pages 221-231, 1980.

[21] Nisha Patel and Chhabikiran Sao "A survey on: visual enhancement and restoration of sub-aqua image", International Journal of Advance Research in Computer and Communication Engineering (IJARCCE), Vol. 3, Issue 2 February 2014.

[22] Harpoonamdeep Kaur and Dr. Rajiv Mahajan "A Review on various Visibility Restoration Techniques, International Journal of Advance Research in Computer and Communication Engineering (IJARCCE), Vol. 3 , Issue 5 May 2014.

[23] F. Cozman and E. Krotkov, "Depth from scattering," in Proc. IEEE Computer Society Conf. Computer Vision and Pattern Recognition, San Juan, PR, 1997, pp. 801806.

[24] Luz Abril Torres-Méndez and Gregory Dudek, "Color Correction of Underwater Images for Aquatic Robot Inspection" Lecture Notes in Computer Science 3757 Springer A. Rangarajan, B.C Vemuri, A.L. Yuille (Eds.), pp. 60-73, ISBN:3-540- 30287-5,2005.

[25] Y. Schechner and N. Karpel "Recovery of underwater visibility and structure by polarization analysis" IEEE JOE, vol. 30, no. 3, pp. 570-587, 2005.

[26] D. He and G. Seet "Divergent-beam lidar imaging in turbid water" Optics and Lasers in Engineering, vol. 41, no. 1, pp. 217-231, 2004.

[27] S. Narasimhan and S. Nayar "Contrast restoration of weather degraded images" IEEE TPAMI, vol. 25 , no. 6 , pp. 713-724, 2003.

[28] E. Nascimento, M. Campos and W. Barros "Stereo based structure recovery of underwater scenes from automatically restored images" SIBGRAPI, pp. 330-337, 2009.

[29] J. Queiroz-Neto, R. Carceroni, W. Barros and M Campos "Underwater stereo" SIBGRAPI, pp. 170-177, 2004

[30] Gaurav Goel, Maitreyee Dutta and Sudhir Goswami Paper Titled "An Approach for Shallow Underwater Images Visibility and Color Improvement" In Indian Journal of Science and Technology, Vol 8 , Issue 35 December, 2015, pp 1-5.

[31] Gaurav Goel, Tahir Ali, Praveen Kr Mishra and Pooja Agarwal Paper Titled "The Comparative Analysis of Visibility Enhancement Techniques for Outdoor Hazy Images and Underwater Images" In International Journal for Research in Emerging Science and Technology, Vol 2, Issue 10, October, 2015, pp 6-11.

[32] Praveen Kr Mishra, Ramakant Verma, Gaurav Goel and Pooja Agarwal Paper Titled "The Conceptual Review of Visibility Refinement Techniques for Outdoor and Night Hazy Images" In International Journal for Research in Emerging Science and Technology, Vol 2, Issue, 10 October, 2015, pp 1-5.

[33 Praveen Kumar Mishra, Maitreyee Dutta Paper Titled "A Scheme for Increasing Visibility of Single Hazy Image under Night Condition" In Indian Journal of Science and Technology, Vol 8, Issue 36 December, 2015, pp 1 
International Journal of Computer Applications (0975 - 8887) Volume 182 - No. 26, November- 2018 